\title{
Partial characterizations of clique-perfect graphs II: diamond-free and Helly circular-arc graphs
}

\author{
Flavia Bonomo a, ${ }^{\mathrm{a}, 1}$ Maria Chudnovsky ${ }^{\mathrm{b}, 2}$ and Guillermo Durán ${ }^{\mathrm{c}, 3}$ \\ ${ }^{a}$ Departamento de Matemática, Facultad de Ciencias Exactas y Naturales, \\ Universidad de Buenos Aires, Buenos Aires, Argentina. \\ b Department of Mathematics, Princeton University, NJ, USA. \\ ${ }^{\mathrm{c}}$ Departamento de Ingeniería Industrial, Facultad de Ciencias Físicas y \\ Matemáticas, Universidad de Chile, Santiago, Chile.
}

\begin{abstract}
A clique-transversal of a graph $G$ is a subset of vertices that meets all the cliques of $G$. A clique-independent set is a collection of pairwise vertex-disjoint cliques. A graph $G$ is clique-perfect if the sizes of a minimum clique-transversal and a maximum clique-independent set are equal for every induced subgraph of $G$. The list of minimal forbidden induced subgraphs for the class of clique-perfect graphs is not known. Another open question concerning clique-perfect graphs is the complexity of the recognition problem. Recently we were able to characterize clique-perfect graphs by a restricted list of forbidden induced subgraphs when the graph belongs to two different subclasses of claw-free graphs. These characterizations lead to polynomial time recognition of clique-perfect graphs in these classes of graphs. In this paper we solve the characterization problem in two new classes of graphs: diamond-free and Helly circular-arc $(H C A)$ graphs. This last characterization leads to a polynomial time recognition algorithm for clique-perfect $H C A$ graphs.
\end{abstract}

Key words: Clique-perfect graphs, diamond-free graphs, Helly circular-arc graphs, K-perfect graphs, perfect graphs. 


\section{Introduction}

Let $G$ be a simple finite undirected graph, with vertex set $V(G)$ and edge set $E(G)$. Denote by $\bar{G}$, the complement of $G$. Given two graphs $G$ and $G^{\prime}$ we say that $G$ contains $G^{\prime}$ if $G^{\prime}$ is isomorphic to an induced subgraph of $G$. When we need to refer to the non-induced subgraph containment relation, we will say so explicitly.

A class of graphs $\mathcal{C}$ is hereditary if for every $G \in \mathcal{C}$, every induced subgraph of $G$ also belongs to $\mathcal{C}$.

The neighborhood of a vertex $v$ is the set $N(v)$ consisting of all the vertices which are adjacent to $v$. The closed neighborhood of $v$ is $N[v]=N(v) \cup\{v\}$. A vertex $v$ of $G$ is universal if $N[v]=V(G)$. Two vertices $v$ and $w$ are twins if $N[v]=N[w]$; and $u$ dominates $v$ if $N[v] \subseteq N[u]$. For an induced subgraph $H$ of $G$ and a vertex $v$ in $V(G) \backslash V(H)$, the set of neighbors of $v$ in $H$ is the set $N(v) \cap V(H)$.

A complete set or just a complete of $G$ is a subset of vertices pairwise adjacent. A clique is a complete set not properly contained in any other. We may also use the term clique to refer to the corresponding complete subgraph. A stable set in a graph $G$ is a subset of pairwise non-adjacent vertices of $G$.

A family of sets $S$ is said to satisfy the Helly property if every subfamily of it, consisting of pairwise intersecting sets, has a common element.

Consider a finite family of non-empty sets. The intersection graph of this family is obtained by representing each set by a vertex, two vertices being adjacent if and only if the corresponding sets intersect.

A circular-arc graph is the intersection graph of arcs of the unit circle. A representation of a circular-arc graph is a collection of circular intervals (of the unit circle), each corresponding to a unique vertex of the graph, such that two intervals intersect if and only if the corresponding vertices are adjacent. A Helly

Email addresses: fbonomo@dc.uba.ar (Flavia Bonomo), mchudnov@Math.Princeton.EDU (Maria Chudnovsky), gduran@dii.uchile.cl (Guillermo Durán).

1 Partially supported by UBACyT Grant X184, PICT ANPCyT Grant 11-09112 and PID Conicet Grant 644/98, Argentina and CNPq under PROSUL project Proc. 490333/2004-4, Brazil.

2 This research was conducted during the period the author served as a Clay Mathematics Institute Research Fellow.

3 Partially supported by FONDECyT Grant 1050747 and Millennium Science Nucleus "Complex Engineering Systems", Chile and CNPq under PROSUL project Proc. 490333/2004-4, Brazil. 
circular-arc $(H C A)$ graph is a circular-arc graph admitting a representation whose arcs satisfy the Helly property. In particular, in a Helly circular-arc representation of a graph, for every clique there is a point of the circle that belongs to the circular intervals corresponding to the vertices in the clique, and to no others. We call such a point an anchor of the clique (please note that an anchor may not be unique).

A graph is clique-Helly $(\mathrm{CH})$ if its cliques satisfy the Helly property, and it is hereditary clique-Helly $(\mathrm{HCH})$ if $H$ is clique-Helly for every induced subgraph $H$ of $G$.

Let $G$ be a graph and $H$ a subgraph of $G$ (not necessarily induced). The graph $H$ is a clique subgraph of $G$ if every clique of $H$ is a clique of $G$.

A complete graph on $n$ vertices is denoted by $K_{n}$. A diamond is the graph isomorphic to $K_{4} \backslash\{e\}$, where $e$ is an edge of $K_{4}$. A graph is diamond-free if it does not contain a diamond.

A claw is the graph isomorphic to $K_{1,3}$. A graph is claw-free if it does not contain a claw. The line graph $L(G)$ of $G$ is the intersection graph of the edges of $G$. A graph $F$ is a line graph if there exists a graph $H$ such that $L(H)=F$. Clearly, line graphs are a subclass of claw-free graphs.

A hole is a chordless cycle of length $n \geq 4$, and it is denoted by $C_{n}$. An antihole is the complement of a hole. A hole or antihole on $n$ vertices is said to be odd if $n$ is odd. A 4-wheel is a graph on five vertices $v_{1}, \ldots, v_{5}$, such that $v_{1} v_{2} v_{3} v_{4} v_{1}$ is a hole and $v_{5}$ is adjacent to all of $v_{1}, v_{2}, v_{3}, v_{4}$.

A clique cover of a graph $G$ is a subset of cliques covering all the vertices of $G$. The clique covering number of $G$, denoted by $k(G)$, is the cardinality of a minimum clique cover of $G$. An obvious lower bound is the maximum cardinality of the stable sets of $G$, the stability number of $G$, denoted by $\alpha(G)$. A graph $G$ is perfect if $\alpha(H)=k(H)$ for every induced subgraph $H$ of $G$. It has been proved recently that a graph $G$ is perfect if and only if no induced subgraph of $G$ is an odd hole or an odd antihole [11], and that perfect graphs can be recognized in polynomial time [10].

The clique graph $K(G)$ of $G$ is the intersection graph of the cliques of $G$. A graph $G$ is $K$-perfect if $K(G)$ is perfect.

A clique-transversal of a graph $G$ is a subset of vertices that meets all the cliques of $G$. A clique-independent set is a collection of pairwise vertex-disjoint cliques. The clique-transversal number and clique-independence number of $G$, denoted by $\tau_{c}(G)$ and $\alpha_{c}(G)$, are the sizes of a minimum clique-transversal and a maximum clique-independent set of $G$, respectively. It is easy to see that $\tau_{c}(G) \geq \alpha_{c}(G)$ for any graph $G$. A graph $G$ is clique-perfect if $\tau_{c}(H)=\alpha_{c}(H)$ 
for every induced subgraph $H$ of $G$. Say that a graph is clique-imperfect when it is not clique-perfect. Clique-perfect graphs have been implicitly studied quite extensively, but the terminology "clique-perfect" has been introduced in [16]. Some known classes of clique-perfect graphs are dually chordal graphs [8], comparability graphs [1] and balanced graphs [3].

The list of minimal forbidden induced subgraphs for the class of clique-perfect graphs is not known. Another open question concerning clique-perfect graphs is the complexity of the recognition problem.

There are some partial results in this direction. In [17], clique-perfect graphs are characterized by minimal forbidden subgraphs for the class of chordal graphs. In [18], minimal graphs $G$ with $\alpha_{c}(G)=1$ and $\tau_{c}(G)>1$ are explicitly described. In [5], clique-perfect graphs are characterized by minimal forbidden subgraphs for two subclasses of claw-free graphs. These characterizations lead to polynomial algorithms for recognizing clique-perfect graphs in these subclasses.

In this paper, we characterize diamond-free clique-perfect graphs by a list of non minimal forbidden subgraphs. Moreover, we give a characterization of clique-perfect graphs for the whole class of Helly circular-arc graphs by minimal forbidden subgraphs. As a corollary of this characterization we can find a polynomial time recognition algorithm for clique-perfect $H C A$ graphs.

Preliminary results of this paper appeared in $[4,6]$.

\section{New families and partial characterizations}

In this section we introduce various families of clique-imperfect graphs, needed to characterize diamond-free and $H C A$ clique-perfect graphs by forbidden subgraphs.

A sun (or trampoline) is a chordal graph $G$ on $2 r$ vertices whose vertex set can be partitioned into two sets, $W=\left\{w_{1}, \ldots, w_{r}\right\}$ and $U=\left\{u_{1}, \ldots, u_{r}\right\}$, such that $W$ is a stable set and for each $i$ and $j, w_{j}$ is adjacent to $u_{i}$ if and only if $i=j$ or $i \equiv j+1(\bmod r)$. A sun is odd if $r$ is odd. A sun is complete if $U$ is a complete.

A generalized sun is defined as follows. Let $G$ be a graph and $C$ be a cycle of $G$ not necessarily induced. An edge of $C$ is non proper (or improper) if it forms a triangle with some vertex of $C$. An $r$-generalized sun, $r \geq 3$, is a graph $G$ whose vertex set can be partitioned into two sets: a cycle $C$ of $r$ vertices, with all its non proper edges $\left\{e_{j}\right\}_{j \in J}$ ( $J$ is permitted be an empty set) and a 
stable set $U=\left\{u_{j}\right\}_{j \in J}$, such that for each $j \in J, u_{j}$ is adjacent only to the endpoints of $e_{j}$. An $r$-generalized sun is said to be odd if $r$ is odd. Clearly odd holes and odd suns are odd generalized suns.

Theorem 1 [7] Odd generalized suns and antiholes of length $t=1,2 \bmod 3$ $(t \geq 5)$ are not clique-perfect.

Unfortunately, not every odd generalized sun is minimally clique-imperfect (with respect to taking induced subgraphs). Nevertheless, odd holes and complete odd suns are minimally clique-imperfect, and we will distinguish other two kinds of minimally clique-imperfect odd generalized suns in order to state a characterization of $H C A$ clique-perfect graphs by minimal forbidden induced subgraphs.

A viking is a graph $G$ such that $V(G)=\left\{a_{1}, \ldots, a_{2 k+1}, b_{1}, b_{2}\right\}, k \geq 2, a_{1} \ldots$ $a_{2 k+1} a_{1}$ is a cycle with only one chord $a_{2} a_{4} ; b_{1}$ is adjacent to $a_{2}$ and $a_{3} ; b_{2}$ is adjacent to $a_{3}$ and $a_{4}$, and there are no other edges in $G$.

A 2-viking is a graph $G$ such that $V(G)=\left\{a_{1}, \ldots, a_{2 k+1}, b_{1}, b_{2}, b_{3}\right\}, k \geq 2$, $a_{1} \ldots a_{2 k+1} a_{1}$ is a cycle with only two chords, $a_{2} a_{4}$ and $a_{3} a_{5} ; b_{1}$ is adjacent to $a_{2}$ and $a_{3} ; b_{2}$ is adjacent to $a_{3}$ and $a_{4} ; b_{3}$ is adjacent to $a_{4}$ and $a_{5}$, and there are no other edges in $G$.

Proposition 2 Vikings and 2-vikings are clique-imperfect.

PROOF. They are odd generalized suns, where in both cases the odd cycle is $a_{1} \ldots a_{2 k+1} a_{1}$, and the stable sets are $\left\{b_{1}, b_{2}\right\}$ and $\left\{b_{1}, b_{2}, b_{3}\right\}$, respectively.

Next we introduce two new families (which are not odd generalized suns or antiholes) of minimal clique-imperfect graphs.

Define the graph $S_{k}, k \geq 2$, as follows: $V\left(S_{k}\right)=\left\{a_{1}, \ldots, a_{2 k+1}, b_{1}, b_{2}, b_{3}\right\}, a_{1}$ $\ldots a_{2 k+1} a_{1}$ is a cycle with only one chord $a_{3} a_{5} ; b_{1}$ is adjacent to $a_{1}$ and $a_{2} ; b_{2}$ is adjacent to $a_{4}$ and $a_{5} ; b_{3}$ is adjacent to $a_{1}, a_{2}, a_{3}$ and $a_{4}$, and there are no other edges in $S_{k}$.

Define the graph $T_{k}, k \geq 2$, as follows: $V\left(T_{k}\right)=\left\{a_{1}, \ldots, a_{2 k+1}, b_{1}, \ldots, b_{5}\right\}$, $a_{1} \ldots a_{2 k+1} a_{1}$ is a cycle with only two chords, $a_{2} a_{4}$ and $a_{3} a_{5} ; b_{1}$ is adjacent to $a_{1}$ and $a_{2} ; b_{2}$ is adjacent to $a_{1}, a_{2}$ and $a_{3} ; b_{3}$ is adjacent to $a_{1}, a_{2}, a_{3}, a_{4}, b_{2}$ and $b_{4} ; b_{4}$ is adjacent to $a_{3}, a_{4}$ and $a_{5} ; b_{5}$ is adjacent to $a_{4}$ and $a_{5}$, and there are no other edges in $T_{k}$.

Proposition 3 Let $k \geq 2$. Then $S_{k}$ and $T_{k}$ are clique-imperfect. 
PROOF. Every clique of $S_{k}$ contains at least two vertices of $a_{1}, \ldots, a_{2 k+1}$, so $\alpha_{c}\left(S_{k}\right) \leq k$. The same holds for $T_{k}$, so $\alpha_{c}\left(T_{k}\right) \leq k$. On the other hand, consider in $S_{k}$ the family of cliques $\left\{a_{1}, a_{2}, b_{1}\right\},\left\{a_{2}, a_{3}, b_{3}\right\},\left\{a_{3}, a_{4}, b_{3}\right\},\left\{a_{4}, a_{5}, b_{2}\right\}$ and either $\left\{a_{5}, a_{1}\right\}$, if $k=2$, or $\left\{a_{5}, a_{6}\right\}, \ldots,\left\{a_{2 k+1}, a_{1}\right\}$, if $k>2$. No vertex of $S_{k}$ belongs to more than two of these $2 k+1$ cliques, so $\tau_{c}\left(S_{k}\right) \geq k+1$. Analogously, consider in $T_{k}$ the family of cliques $\left\{a_{1}, a_{2}, b_{1}\right\},\left\{a_{2}, a_{3}, b_{2}, b_{3}\right\},\left\{a_{3}, a_{4}, b_{3}, b_{4}\right\}$, $\left\{a_{4}, a_{5}, b_{5}\right\}$ and either $\left\{a_{5}, a_{1}\right\}$, if $k=2$, or $\left\{a_{5}, a_{6}\right\}, \ldots,\left\{a_{2 k+1}, a_{1}\right\}$, if $k>2$. No vertex of $T_{k}$ belongs to more than two of these $2 k+1$ cliques, so $\tau_{c}\left(T_{k}\right) \geq$ $k+1$.

The minimality of vikings, 2 -vikings, $S_{k}$ and $T_{k}(k \geq 2)$ will be proved as a corollary of the main theorem of Section 4.

In [18] the minimal graphs $G$ such that $K(G)$ is complete (i.e. $\alpha_{c}(G)=1$ ) and no vertex of $G$ is universal (i.e. $\tau_{c}(G)>1$ ) are characterized. The graph $Q_{n}$, $n \geq 3$, is defined as follows: $V\left(Q_{n}\right)=\left\{u_{1}, \ldots, u_{n}\right\} \cup\left\{v_{1}, \ldots, v_{n}\right\}$ is a set of $2 n$ vertices; $v_{1}, \ldots, v_{n}$ induce $\overline{C_{n}}$; for each $1 \leq i \leq n, N\left[u_{i}\right]=V\left(Q_{n}\right)-\left\{v_{i}\right\}$.

The following result will be useful to us:

Theorem 4 [18] For $k \geq 1, \alpha_{c}\left(Q_{2 k+1}\right)=1$ and $\tau_{c}\left(Q_{2 k+1}\right)=2$. Moreover, if $G$ is a graph such that $\alpha_{c}(G)=1$ and $\tau_{c}(G)>1$, then $G$ contains $Q_{2 k+1}$ for some $k \geq 1$.

For some classes of graphs, it is enough to exclude some odd generalized suns and some antiholes in order to guarantee that the graph is clique-perfect:

Theorem 5 [17] Let $G$ be a chordal graph. Then $G$ is clique-perfect if and only if no induced subgraph of $G$ is an odd sun.

Theorem 6 [5] Let $G$ be a line graph. Then $G$ is clique-perfect if and only if no induced subgraph of $G$ is an odd hole or a 3-sun.

Theorem 7 [5] Let $G$ be an $H C H$ claw-free graph. Then $G$ is clique-perfect if and only if no induced subgraph of $G$ is an odd hole or an antihole of length seven.

A similar result holds for diamond-free graphs. This, however, is not the case for $H C A$ graphs. The graphs $S_{k}$ and $T_{k}$ are minimal clique-imperfect $H C A$ graphs; but these are the only minimal clique-imperfect $H C A$ graphs which are not odd generalized suns or antiholes.

Our main results are the following two theorems:

Theorem 8 Let $G$ be a diamond-free graph. Then $G$ is clique-perfect if and 
only if no induced subgraph of $G$ is an odd generalized sun.

Theorem 9 Let $G$ be an HCA graph. Then $G$ is clique-perfect if and only if it does not contain a 3-sun, an antihole of length seven, an odd hole, a viking, a 2-viking or one of the graphs $S_{k}$ or $T_{k}$.

In the next two sections we prove Theorems 8 and 9 .

\section{Diamond-free graphs}

The following lemma establishes a connection between the parameters involved in the definition of clique-perfect graphs and those corresponding to perfect graphs.

Lemma 10 [7] Let $G$ be a graph. Then:

(1) $\alpha_{c}(G)=\alpha(K(G))$.

(2) $\tau_{c}(G) \geq k(K(G))$. Moreover, if $G$ is clique-Helly, then $\tau_{c}(G)=k(K(G))$.

Hereditary clique-Helly graphs are of particular interest because in this case it follows from Lemma 10 that if $K(H)$ is perfect for every induced subgraph $H$ of $G$, then $G$ is clique-perfect (the converse is not necessarily true).

The class of hereditary clique-Helly graphs can be characterized by forbidden induced subgraphs.

Theorem 11 [19] A graph $G$ is hereditary clique-Helly if and only if it does not contain the graphs of Figure 1.
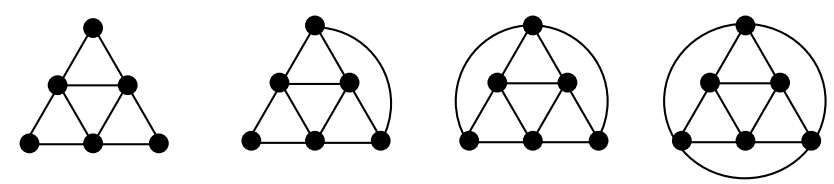

Fig. 1. Forbidden induced subgraphs for hereditary clique-Helly graphs: (left to right) 3-sun (or 0-pyramid), 1-pyramid, 2-pyramid and 3-pyramid.

As a direct corollary of this characterization, it follows that diamond-free graphs are $\mathrm{HCH}$.

The following is a useful fact about hereditary clique-Helly graphs:

Proposition 12 Let $\mathcal{L}$ be a hereditary graph class, which is $\mathrm{HCH}$ and such that every graph in $\mathcal{L}$ is K-perfect. Then every graph in $\mathcal{L}$ is clique-perfect. 
PROOF. Let $G$ be a graph in $\mathcal{L}$. Let $H$ be an induced subgraph of $G$. Since $\mathcal{L}$ is hereditary, $H$ is a graph in $\mathcal{L}$, so it is K-perfect. Since $\mathcal{L}$ is an $H C H$ class, $H$ is clique-Helly and then, by Lemma $10, \alpha_{C}(H)=\alpha(K(H))=k(K(H))=$ $\tau_{C}(H)$, and the result follows.

We can now prove the main result of this section.

Proof of Theorem 8. By Theorem 1, if $G$ is clique-perfect then no induced subgraph of $G$ is an odd generalized sun. Let us prove the converse. Let $G$ be a diamond-free graph such that no induced subgraph of $G$ is an odd generalized sun.

First we show that $K(G)$ contains no odd holes or odd antiholes, and therefore it is perfect. By [9], $G$ being diamond-free implies that $K(G)$ is diamond-free, and hence $K(G)$ contains no antihole of length at least 7. Suppose $K(G)$ contains an odd hole $k_{1} k_{2} \ldots k_{2 n+1}$, where $k_{1}, \ldots, k_{2 n+1}$ are cliques of $G$. Then $G$ contains an odd cycle $v_{1} v_{2} \ldots v_{2 n+1} v_{1}$, where $v_{i}$ belongs to $k_{i} \cap k_{i+1}$ and no other $k_{j}$. Since $G$ contains no odd generalized suns, we may assume that some edge of this cycle, say, $v_{1} v_{2}$ is in a triangle with another vertex of the cycle, say $v_{m}$. Now $v_{1}, v_{2}$ both belong to $k_{2}$, and $v_{m}$ does not. Since $k_{2}$ is a clique, it follows that $v_{m}$ has a non-neighbor $w$ in $k_{2}$. But now $\left\{v_{1}, v_{2}, v_{m}, w\right\}$ induces a diamond, a contradiction. Finally, Proposition 12 completes the proof.

\section{Helly circular-arc graphs}

The main result of this section is the following: if a graph $G$ is $H C A$, then $G$ is clique-perfect if and only if it does not contain the graphs of Figure 2. (This is Theorem 9.)

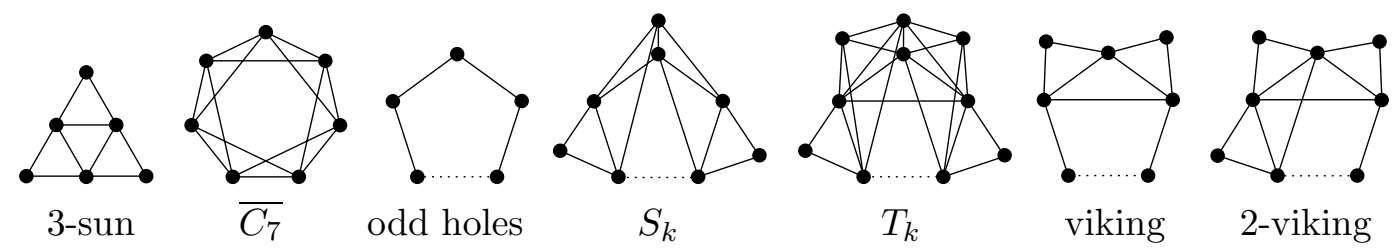

Fig. 2. Minimal forbidden subgraphs for clique-perfect graphs inside the class of $H C A$ graphs. Dotted lines replace any induced path of odd length at least 1.

In fact, we show that an $H C A$ graph that does not contain any of the graphs of Figure 2 is K-perfect. In general, the class of clique-perfect graphs is neither a subclass nor a superclass of the class of K-perfect graphs. But the K-perfection allows us to use arguments similar to those used in the proof of Proposition 12, in order to prove Theorem 9 for $H C A$ graphs that are also $H C H$. The graphs in $H C A \backslash H C H$ are handled separately. 
We start with some easy results about $H C H$ and $H C A$ graphs.

Theorem 13 [7] Let $G$ be an $H C H$ graph such that $K(G)$ is not perfect.

(1) If $K(G)$ contains $\overline{C_{7}}$ as induced subgraph, then $G$ contains a clique subgraph $H$ in which identifying twin vertices and then removing dominated vertices we obtain $\overline{C_{7}}$, and such that $K(H)=\overline{C_{7}}$.

(2) If $K(G)$ contains $C_{2 k+1}$ as induced subgraph, for some $k \geq 2$, then $G$ contains a clique subgraph $H$ in which identifying twin vertices and then removing dominated vertices we obtain $C_{2 k+1}$, and such that $K(H)=$ $C_{2 k+1}$.

In this section we will call a sector an arc of a circle defined by two points, in order to distinguish them from arcs corresponding to vertices of an $H C A$ graph. For example, in Figure 3, the bold arc is one of the two sectors defined by the points $a$ and $b$. Given a collection $\mathcal{C}$ of points on the circle, for $a, b, c \in \mathcal{C}$ we say that $c$ is $\mathcal{C}$-between $a$ and $b$ if the sector defined by $a$ and $b$ that contains $c$ does not contain any other point of $\mathcal{C}$. For example, in Figure $3, c$ is $\{a, b, c, d, e\}$-between $a$ and $b$ but $d$ is not.

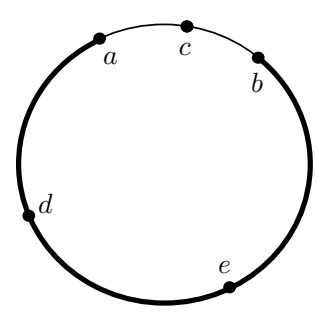

Fig. 3. Example of notation. The bold arc is one of the two sectors defined by the points $a$ and $b$ of the circle. $c$ is $\{a, b, c, d, e\}$-between $a$ and $b$ but $d$ is not.

Lemma 14 Let $G$ be an $H C A$ graph that has an $H C A$ representation with no two arcs covering the circle. Then $\mathrm{G}$ is $\mathrm{HCH}$.

PROOF. Suppose not. By Theorem 11, G contains a 0-,1-, 2-, or 3-pyramid $P$. Let $\left\{v_{1}, \ldots, v_{6}\right\}$ be the vertices of $P$, such that $v_{1}, v_{2}, v_{3}$ form a triangle; $v_{4}$ is adjacent to $v_{2}$ and $v_{3}$ but not to $v_{1} ; v_{5}$ is adjacent to $v_{1}$ and $v_{3}$ but not to $v_{2} ; v_{6}$ is adjacent to $v_{1}$ and $v_{2}$ but not to $v_{3}$. Since $P$ is an induced subgraph of $G, P$ has an $H C A$ representation with no two arcs covering the circle. Let $\mathcal{A}=\left\{A_{i}\right\}_{1 \leq i \leq 6}$ be such a representation, where the arc $A_{i}$ corresponds to the vertex $v_{i}$. The sets $C_{1}=\left\{v_{1}, v_{2}, v_{3}\right\}$ and $C_{2}=\left\{v_{1}, v_{2}, v_{6}\right\}$ are cliques of $P$, let $a$ be an anchor of $C_{1}$ and $b$ of $C_{2}$. Then $a$ and $b$ are distinct points of the circle. Let $S_{1}$ and $S_{2}$ be the two sectors with ends $a, b$. Since $A_{1}, A_{2}$ do not cover the circle, and $a, b$ belong to both $A_{1}$ and $A_{2}$, we may assume that $S_{1}$ is included both in $A_{1}$ and in $A_{2}$. Since $a \in A_{3}$ but $b \notin A_{3}$, it follows that $A_{3}$ has an endpoint, say $c$, in $S_{1} \backslash\{b\}$ (see Figure 4). But now, since the pairs $A_{1}, A_{3}$ and $A_{2}, A_{3}$ do not cover the circle, it follows that either 
$A_{1} \cap A_{3} \subseteq A_{2}$, or $A_{2} \cap A_{3} \subseteq A_{1}$. In the former case there is no anchor for the clique $\left\{v_{1}, v_{3}, v_{5}\right\}$, and in the later there is none for the clique $\left\{v_{2}, v_{3}, v_{4}\right\}$; in both cases a contradiction.

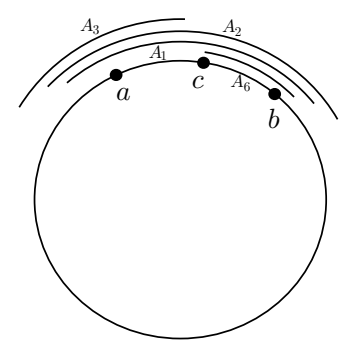

Fig. 4. Scheme of representation of $\operatorname{arcs} A_{6}, A_{1}, A_{2}$ and $A_{3}$, in the proof of Lemma 14 .

Lemma 15 Every HCA representation of a 4-wheel has two arcs covering the circle.

PROOF. Let $a_{1}, a_{2}, a_{3}, a_{4}, b$ be the vertices of a 4 -wheel $W$, where $a_{1} a_{2} a_{3} a_{4} a_{1}$ is a cycle of length four and $b$ is adjacent to all of $a_{1}, a_{2}, a_{3}, a_{4}$, and let $\mathcal{A}=\left\{A_{1}\right.$, $\left.A_{2}, A_{3}, A_{4}, B\right\}$ be an $H C A$ representation of $W$. Let $p_{1}, p_{2}, p_{3}$ and $p_{4}$ be anchors of the cliques $\left\{a_{1}, a_{2}, b\right\},\left\{a_{2}, a_{3}, b\right\},\left\{a_{3}, a_{4}, b\right\},\left\{a_{4}, a_{1}, b\right\}$, respectively. Then there are only two possible circular orders of the anchors: $p_{1}, p_{2}, p_{3}, p_{4}$ and the reverse one, and for $1 \leq i \leq 4$, each arc $A_{i}$ passes exactly through $p_{i}$ and $p_{i-1}$ (index operations are done modulo 4 ). Since the arc $B$ passes through the four points $p_{i}$, it follows that $B$ and one of the $A_{i}$ cover the circle.

Lemma 16 Let $G$ be an $H C A$ graph and let $\mathcal{A}$ be an $H C A$ representation of $G$, such that no two arcs of $\mathcal{A}$ cover the circle. Then no three arcs of $\mathcal{A}$ cover the circle.

PROOF. Let $\mathcal{C}$ denote the unit circle. Suppose that there are three $\operatorname{arcs} A$, $B$, and $C$ in $\mathcal{A}$ covering $\mathcal{C}$. Since $A \cup B$ does not cover $\mathcal{C}$, there is a point $c$ in $\mathcal{C} \backslash(A \cup B)$. Since $\mathcal{C}=A \cup B \cup C$, it follows that $c \in C$. Analogously, there exist points $a$ and $b$ in $A \backslash(B \cup C)$ and $B \backslash(A \cup C)$, respectively. Since the arcs are open, $\mathcal{C}=A \cup B \cup C$, and the union of any two of $A, B, C$ does not include $\mathcal{C}$, it follows that $A \cap B, A \cap C$ and $B \cap C$ are all non-empty. Since $\mathcal{A}$ satisfies the Helly property, there exists a point $p \in A \cap B \cap C$. But since $a \in A \backslash(B \cup C)$, and $b, c \notin A$, it follows that $p$ does not lie $\{a, b, c\}$-between $b$ and $c$. Similarly, $p$ does not lie $\{a, b, c\}$-between $a$ and $b$ or $\{a, b, c\}$-between $a$ and $c$, a contradiction.

Lemma 17 Let $S$ denote the unit circle. Let $G$ be an $H C A$ graph that has an $H C A$ representation with no two arcs covering $S$, and let $\mathcal{A}$ be such a 
representation. Let $H$ be a clique subgraph of $G$. Then $H$ is $H C A$ and has an $H C A$ representation $\mathcal{A}^{\prime}$ with no two arcs covering $S$. Moreover, let $M_{1}, \ldots, M_{s}$ be the cliques of $H$, and for $1 \leq i \leq s$ let $a_{i}$ be an anchor of $M_{i}$ in $\mathcal{A}$. Let $\varepsilon=\frac{1}{3} \min _{1 \leq i<j \leq s} \operatorname{dist}\left(a_{i}, a_{j}\right)$, where $\operatorname{dist}\left(a_{i}, a_{j}\right)$ denotes the length of the shortest sector of $S$ between $a_{i}$ and $a_{j}$. For an arc $A \in \mathcal{A}$ that contains at least one of the points $a_{1}, \ldots, a_{s}$, let the derived arc $A^{\prime}$ of $A$ be defined as follows: let $a_{i_{k}}, \ldots, a_{i_{m}}$ be the points of $a_{1}, \ldots, a_{s}$ traversed by $A$ in clockwise order, let $u$ be the point of $S$ which is at distance $\varepsilon$ from $a_{i_{k}}$ going anti-clockwise, and $v$ the point of $S$ which is at distance $\varepsilon$ from $a_{i_{m}}$ going clockwise. Then $A^{\prime}$ is the arc with endpoints $u$ and $v$ and containing all of $a_{i_{k}}, \ldots, a_{i_{m}}$. In this notation, $\mathcal{A}^{\prime}$ is precisely the set of all arcs $A^{\prime}$ that are the derived arcs of some $A \in \mathcal{A}$ such that $A$ contains at least one of $a_{1}, \ldots, a_{s}$. Please note that $\mathcal{A}^{\prime}$ depends on the choice of the anchors $a_{1}, \ldots, a_{s}$.

PROOF. Let $H^{\prime}$ be the intersection graph of the arcs of $\mathcal{A}^{\prime}$. We claim that $H^{\prime}$ is isomorphic to $H$. Since the arcs of $\mathcal{A}^{\prime}$ are sub-arcs of the $\operatorname{arcs}$ of $\mathcal{A}$ that correspond to vertices of $G$ that belong to $\bigcup_{i=1}^{s} M_{i}$, there is a one-to-one correspondence between the vertices of $H^{\prime}$ and the vertices of $H$, and we may assume that $V(H)=V\left(H^{\prime}\right)$. Moreover, for every clique $M_{i}$ and every $A \in \mathcal{A}$, the derived arc of $A$ contains $a_{i}$ if and only if $A$ does. So $M_{1}, \ldots, M_{s}$ are cliques on $H^{\prime}$, and $a_{i}$ is an anchor of $M_{i}$. Since two vertices of a graph are adjacent if and only if there exists a clique containing them both, in order to show that $H$ is isomorphic to $H^{\prime}$, it remains to check that every two adjacent vertices of $H^{\prime}$ belong to $M_{i}$ for some $i$. But it follows from the construction of $\mathcal{A}^{\prime}$ (and in particular from the choice of $\varepsilon$ ) that $A_{1}^{\prime} \cap A_{2}^{\prime} \neq \emptyset$ for $A_{1}^{\prime}, A_{2}^{\prime} \in \mathcal{A}^{\prime}$, if and only if $a_{i} \in A_{1}^{\prime} \cap A_{2}^{\prime}$ for some $1 \leq i \leq s$, which means that the corresponding vertices of $H^{\prime}$ belong to the clique $M_{i}$. This proves that $E(H)=E\left(H^{\prime}\right)$ and completes the proof of the lemma.

An example of the construction of Lemma 17 can be seen in Figure 5.
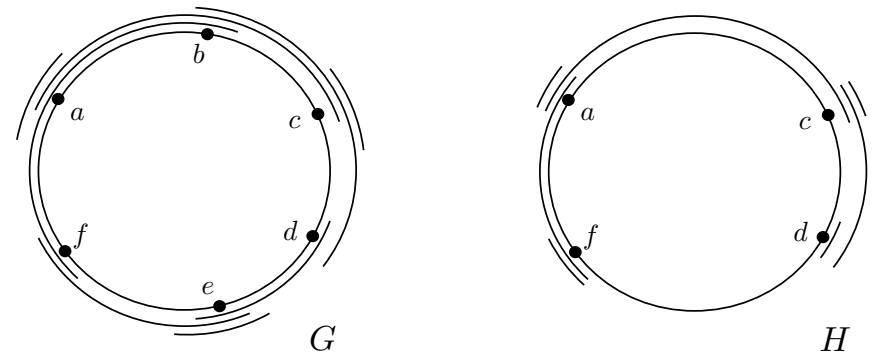

Fig. 5. $H C A$ representation of the clique subgraph $H$ of $G$ whose cliques are $a, c, d$ and $f$.

Remark 18 Let $G$ be an $H C A$ graph with representation $\mathcal{A}$, and let $H$ be a clique subgraph of $G$ with representation $\mathcal{A}^{\prime}$ given by Lemma 17, with anchors 
$a_{1}, \ldots, a_{s}$. Let $A_{1}^{\prime}, A_{2}^{\prime} \in \mathcal{A}^{\prime}$ be the derived arcs of $A_{1}, A_{2} \in \mathcal{A}$. Then $A_{1} \cap A_{2}$ may be non-empty even if $A_{1}^{\prime}, A_{2}^{\prime}$ are disjoint, but no point of $A_{1} \backslash A_{1}^{\prime}$ or $A_{2} \backslash A_{2}^{\prime}$ belongs to $\left\{a_{1}, \ldots, a_{s}\right\}$.

Lemma 19 Let $G$ be an $H C A$ graph and let $\mathcal{A}$ be an $H C A$ representation of $G$. Let $M_{1}, \ldots, M_{k}$, with $k \geq 5$, be a set of cliques of $G$ such that $M_{i} \cap M_{i+1}$ is non-empty for $i=1, \ldots, k$, and $M_{i} \cap M_{j}$ is empty for $j \neq i, i+1, i-1$ (index operations are done modulo $k$ ). Let $S=\left\{v_{1}, \ldots, v_{k}\right\}$ such that $v_{i} \in M_{i-1} \cap M_{i}$. Let $w \in M_{i} \backslash S$ non-adjacent to $v_{i+2}$. Then the neighbors of $w$ in $S$ are either $\left\{v_{i}, v_{i+1}\right\}$, or $\left\{v_{i-1}, v_{i}, v_{i+1}\right\}$, or $\left\{v_{i-2}, v_{i-1}, v_{i}, v_{i+1}\right\}$.

PROOF. For $1 \leq i \leq k$ let $m_{i}$ be an anchor of $M_{i}$, let $A_{i}$ be the arc of $\mathcal{A}$ corresponding to $v_{i}$, and let $W$ be the arc corresponding to $w$. Since for every $i, A_{i}$ contains $m_{i-1}$ and $m_{i}$, and no $m_{j}$ with $j \neq i-1, i$, it follows that there are only two possible circular orders of the anchors: $m_{1}, m_{2}, \ldots, m_{k}$ and the reverse one. Since $w$ belongs to $M_{i}$, it is adjacent to $v_{i}$ and $v_{i+1}$, and $m_{i} \in W$. Since $w$ is non-adjacent to $v_{i+2}, w$ does not belong to $M_{i+1}$, and $m_{i+1} \notin W$. Since $w \in M_{i}$ and $M_{i}$ is disjoint from $M_{j}$ for $j \neq i-1, i, i+1$, it follows that $m_{j} \notin W$ for $j \neq i-1, i$ (see Figure 6 ). Now, if $m_{i-1} \notin W$, then the neighbors of $w$ in $S$ are $v_{i}$ and $v_{i+1}$ or $v_{i-1}, v_{i}, v_{i+1}$, and if $m_{i-1} \in W$, then the neighbors of $w$ in $W$ are $v_{i-1}, v_{i}, v_{i+1}$ or $v_{i-2}, v_{i-1}, v_{i}, v_{i+1}$.

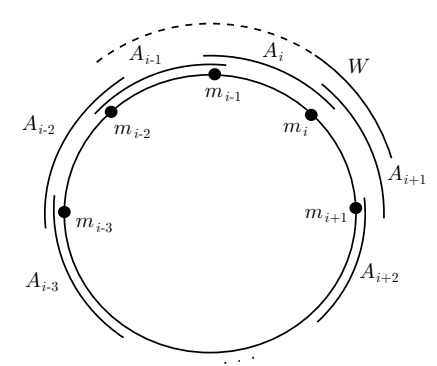

Fig. 6. Scheme of representation of $\operatorname{arcs} A_{i-3}, \ldots, A_{i+2}$ and $W$, in the proof of Lemma 19.

In the next theorem we give a sufficient condition for the clique graph of an $H C A$ graph to be perfect.

Theorem 20 Let $G$ be an HCA graph. If $G$ does not contain any of the graphs in Figure 2, then $K(G)$ is perfect.

PROOF. Let $G$ be an $H C A$ graph which does not contain any of the graphs in Figure 2, and $\mathcal{A}$ be an $H C A$ representation of $G$. Assume first that there are two arcs $A_{1}, A_{2} \in \mathcal{A}$ covering the circle, and let $v_{1}, v_{2}$ be the corresponding vertices of $G$. Then the clique-transversal number of $G$ is at most two, because every anchor of a clique of $G$ is contained in one of $A_{1}, A_{2}$, and therefore every 
clique contains either $v_{1}$ or $v_{2}$. Since, by Lemma 10, the clique covering number of $K(G)$ is less or equal to the clique-transversal number of $G, K(G)$ is the complement of a bipartite graph, and so it is perfect.

So we may assume no two arcs of $\mathcal{A}$ cover the circle, and so by Lemma 16 no three arcs of $\mathcal{A}$ cover the circle. By Lemma 14, $G$ is $H C H$, so $K(G)$ is also $H C H$ [2]. Consequently, if $K(G)$ is not perfect, then it contains an odd hole or $\overline{C_{7}}$ (for every antihole of length at least eight contains a 2-pyramid, and therefore is not $\mathrm{HCH}$ by Theorem 11).

Suppose first that $K(G)$ contains $\overline{C_{7}}$. By Theorem 13, $G$ contains a clique subgraph $H$ in which identifying twin vertices and then removing dominated vertices we obtain $\overline{C_{7}}$. Consider the $H C A$ representation $\mathcal{A}^{\prime}$ of $H$ given by Lemma 17 , and let $v_{1}, \ldots, v_{7}$ be vertices inducing $\overline{C_{7}}$ in $H$, where the cliques are $\left\{v_{1}, v_{3}, v_{5}\right\},\left\{v_{3}, v_{5}, v_{7}\right\},\left\{v_{5}, v_{7}, v_{2}\right\},\left\{v_{7}, v_{2}, v_{4}\right\},\left\{v_{2}, v_{4}, v_{6}\right\},\left\{v_{4}, v_{6}, v_{1}\right\}$ and $\left\{v_{6}, v_{1}, v_{3}\right\}$. That is essentially the unique circular order of the cliques (the other possible order is the reverse one), so the arcs $A_{1}, \ldots, A_{7}$ corresponding to $v_{1}, \ldots, v_{7}$ must appear in $\mathcal{A}^{\prime}$ as in Figure 7 .
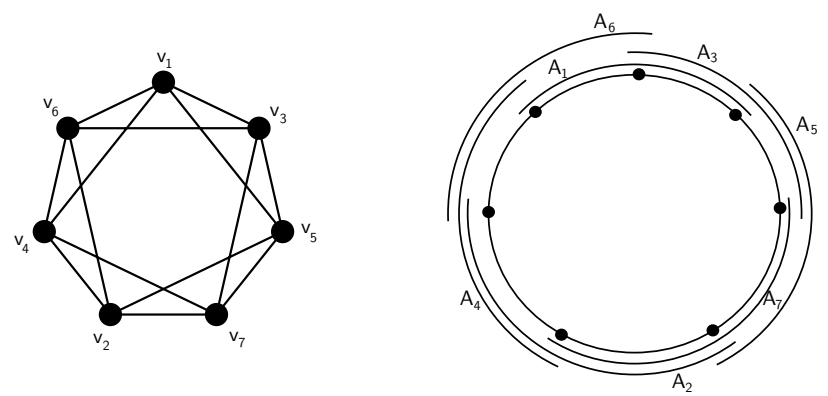

Fig. 7. $H C A$ representation of $\overline{C_{7}}$.

If some pair of non adjacent vertices $v_{i}, v_{j}$ in $H$ are adjacent in $G$, then there are three arcs covering the circle in $\mathcal{A}$, a contradiction. Otherwise $\left\{v_{1}, \ldots, v_{7}\right\}$ induce $\overline{C_{7}}$ in $G$, a contradiction.

Next suppose that $K(G)$ contains $C_{2 k+1}$, for some $k \geq 2$. By Theorem 13, $G$ contains a clique subgraph $H$ in which identifying twin vertices and then removing dominated vertices we obtain $C_{2 k+1}$, and such that $K(H)=C_{2 k+1}$. Consider the $H C A$ representation $\mathcal{A}^{\prime}$ of $H$ given by Lemma 17 corresponding to anchors $a_{1}, \ldots, a_{2 k+1}$, and let $v_{1}, \ldots, v_{2 k+1}$ be vertices inducing $C_{2 k+1}$ in $H$, where the cliques are $v_{i} v_{i+1}$ for $1 \leq i \leq n-1$ and $v_{n} v_{1}$. Then in $G$ the graph induced by $v_{1}, \ldots, v_{2 k+1}$ is a cycle, say $C$, with chords. We assume that $v_{1}, \ldots, v_{2 k+1}$ are chosen to minimize the number $N$ of such chords. Again, that is essentially the unique circular order of the cliques (the other possible order is the reverse one), so the $\operatorname{arcs} A_{1}^{\prime}, \ldots, A_{2 k+1}^{\prime}$ corresponding to $v_{1}, \ldots, v_{2 k+1}$ must appear in $\mathcal{A}^{\prime}$ as in Figure 8.

Now it is possible that two disjoint arcs $A_{i}^{\prime}, A_{j}^{\prime} \in \mathcal{A}^{\prime}$ are derived from arcs 


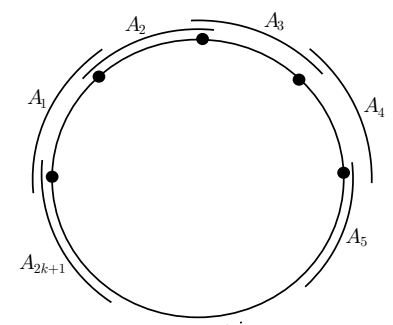

Fig. 8. $H C A$ representation of $C_{2 k+1}, k \geq 2$.

$A_{i}, A_{j} \in \mathcal{A}$ whose intersection is non-empty, but it follows from Remark 18 that in this case $|j-i|=2$ (throughout this proof, indices of vertices in a cycle should be read modulo the length of the cycle). The proof now breaks into cases depending on the values of $k$ and $N$.

Case $k=2$ :

Since there are no three $\operatorname{arcs}$ in $\mathcal{A}$ covering the circle, $C$ has at most one chord incident with each vertex and so $N \leq 2$. The possible $H C A$-representations of the subgraph of $G$ induced by $\left\{v_{1}, \ldots, v_{5}\right\}$ are depicted in Figure 9. Let $M_{1}, \ldots, M_{5}$ be the cliques of $H$ such that $M_{1}$ contains $v_{1}$ and $v_{2}, M_{2}$ contains $v_{2}$ and $v_{3}, \ldots, M_{5}$ contains $v_{5}$ and $v_{1}$, for $1 \leq i \leq 5, a_{i}$ is an anchor of $M_{i}$, and the vertices corresponding to $M_{1}, M_{2}, \ldots, M_{5}$ induce $C_{5}$ in $K(G)$. Let $A=\left\{a_{1}, a_{2}, a_{3}, a_{4}, a_{5}\right\}$.
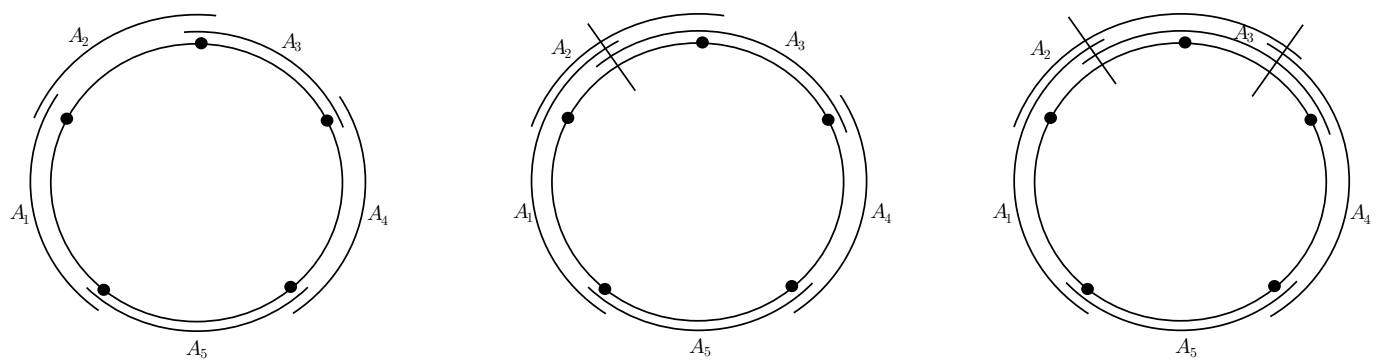

Fig. 9. Possible cases for $k=2$, corresponding to no chords, one chord or two chords in the cycle.

1. $\underline{\mathrm{N}=0}$ : In this case $G$ contains an odd hole, a contradiction.

2. $\underline{\mathrm{N}=1}$ : Suppose that the vertices $v_{1}$ and $v_{3}$ are adjacent in $G$. As $v_{3}$ does not belong to $M_{1}$, there is a vertex $w_{1}$ in $M_{1}$ which is not adjacent to $v_{3}$. Analogously, there is a vertex $w_{2}$ in $M_{2}$ which is not adjacent to $v_{1}$. The vertices $w_{1}$ and $w_{2}$ are non-adjacent, otherwise $v_{1}, v_{3}, w_{2}, w_{1}, v_{2}$ induce a 4 -wheel, which does not have an $H C A$ representation with no two arcs covering the circle (Lemma 15). For $i=1,2, w_{i}$ can have two, three or four neighbors in $C$.

2.1. If $w_{1}$ and $w_{2}$ have two neighbors each one, then $\left\{v_{1}, v_{2}, v_{3}, v_{4}, v_{5}, w_{1}\right.$, $\left.w_{2}\right\}$ induce a viking. 
2.2. If $w_{1}$ and $w_{2}$ have four neighbors each one, then $\left\{v_{1}, w_{2}, w_{1}, v_{3}, v_{5}\right.$, $\left.v_{2}, v_{4}\right\}$ induce $\overline{C_{7}}$.

2.3. If one of $w_{1}, w_{2}$ has three neighbors, say $w_{1}$, for the other case is symmetric, then if follows from Lemma 19 that $w_{1}$ is adjacent to $v_{5}, v_{1}, v_{2}$. But now $\left\{w_{1}, v_{2}, v_{3}, v_{4}, v_{5}\right\}$ induce $C_{5}$.

2.4. If one of $w_{1}, w_{2}$ has two neighbors and the other one has four neighbors, we may assume that $w_{1}$ has two and $w_{2}$ has four (the other case is symmetric). The clique $M_{4}$ does not intersect $M_{2}$, so $w_{2}$ does not belong to $M_{4}$ and there is a vertex $w_{3}$ in $M_{4}$ which is not adjacent to $w_{2}$.

If the arcs corresponding to $w_{3}$ and $v_{3}$ intersect in a point of the circle that is $A$-between $a_{3}$ and $a_{4}$, then one of them passes through a point that belongs both to the arc corresponding to $v_{5}$ and to the arc corresponding to $w_{2}$, but $w_{3}$ is non-adjacent to $w_{2}$ and $v_{3}$ is nonadjacent to $v_{5}$, a contradiction. If the arcs corresponding to $w_{3}$ and $v_{3}$ intersect in a point of the circle $A$-between $a_{1}$ and $a_{2}$, then the arcs corresponding to $v_{3}, v_{4}$ and $w_{3}$ cover the circle. So $w_{3}$ and $v_{3}$ are not adjacent, and $w_{3}$ can be adjacent either to $v_{4}, v_{5}, v_{1}$ and $v_{2}$; or to $v_{4}$, $v_{5}$ and $v_{1}$; or only to $v_{4}$ and $v_{5}$. In the first case, the vertices $v_{1}, w_{2}, w_{3}$, $v_{3}, v_{5}, v_{2}, v_{4}$ induce $\overline{C_{7}}$. In the second case, the vertices $v_{1}, v_{2}, w_{2}, v_{4}, w_{3}$ induce $C_{5}$. In the last case, the eight vertices induce $S_{2}$.

3. $\underline{N=2}$ : The same vertex cannot belong to two chords, so all the cases are symmetric to the case where $v_{1}$ is adjacent to $v_{3}$ and $v_{2}$ to $v_{4}$. As $v_{3}$ does not belong to $M_{1}$, there is a vertex $w_{1}$ in $M_{1}$ which is not adjacent to $v_{3}$. Analogously, as $v_{2}$ does not belong to $M_{3}$, there is a vertex $w_{3}$ in $M_{3}$ which is not adjacent to $v_{2}$.

Please note that if $w_{3}$ is adjacent to $v_{1}$ then their corresponding arcs must intersect in a point of the circle $A$-between $a_{4}$ and $a_{5}$, because $w_{3}$ is not adjacent to $v_{2}$. But in this case the arcs corresponding to $v_{1}, v_{3}$ and $w_{3}$ cover the circle, so $w_{3}$ is not adjacent to $v_{1}$. Analogously, we can prove that $w_{1}$ is not adjacent to $v_{4}$.

3.1. If $w_{1}$ and $w_{3}$ are adjacent, then their corresponding arcs must intersect in a point of the circle $A$-between $a_{4}$ and $a_{5}$, because $w_{1}$ is non-adjacent to $v_{3}$ and $v_{4}$ and $w_{3}$ is non-adjacent to $v_{1}$ and $v_{2}$. So both are adjacent to $v_{5}$, and the vertices $v_{1}, v_{4}, w_{1}, v_{3}, v_{5}, v_{2}, w_{3}$ induce $\overline{C_{7}}$.

3.2. If $w_{1}$ and $w_{3}$ are not adjacent but both of them are adjacent to $v_{5}$, the vertices $w_{1}, v_{2}, v_{3}, w_{3}, v_{5}$ induce $C_{5}$.

3.3. The remaining case is when $w_{1}$ and $w_{3}$ are not adjacent but at most one of them is adjacent to $v_{5}$.

For this case, we have to consider the clique $M_{2}$. Since $v_{1}$ and $v_{4}$ do not belong to $M_{2}$, there is a vertex in $M_{2}$ which is not adjacent to $v_{1}$ and there is a vertex in $M_{2}$ which is not adjacent to $v_{4}$.

3.3.1. If there is a vertex $w$ which is non-adjacent to $v_{1}$ and $v_{4}$, then $w$ 
cannot be adjacent either to $w_{1}$ or $w_{3}$, otherwise $v_{1}, v_{3}, w, w_{1}$, $v_{2}$ (or $v_{2}, w, w_{3}, v_{4}, v_{3}$, respectively) induce a 4 -wheel, a contradiction by Lemma 15 .

Therefore, if each of $w_{1}$ and $w_{3}$ has two neighbors in $C$, then the vertices $v_{1}, \ldots, v_{5}, w_{1}, w, w_{3}$ induce a 2-viking in $G$, and, if $w_{1}$ and $w_{3}$ have two and three neighbors (respectively) in $C$, the vertices $v_{1}, v_{2}, v_{3}, w_{3}, v_{5}, w_{1}, w$ induce a viking in $G$ (the case when $w_{1}$ has three neighbors and $w_{3}$ has two neighbors in $C$ is symmetric).

3.3.2. If there is no such a vertex $w$, every vertex of $M_{2}$ is either adjacent to $v_{1}$ or to $v_{4}$. Then there exist two vertices $w_{2}$ and $w_{4}$ in $M_{2}$, such that $w_{2}$ is adjacent to $v_{4}$ but not to $v_{1}$ and $w_{4}$ is adjacent to $v_{1}$ but not to $v_{4}$. Since by Lemma $15 G$ does not contain a 4 -wheel, it follows that $w_{2}$ is not adjacent to $w_{1}$ and $w_{4}$ is not adjacent to $w_{3}$. If neither $w_{4}$ nor $w_{2}$ is adjacent to $v_{5}$, then the vertices $v_{1}, w_{4}, w_{2}, v_{4}, v_{5}$ induce $C_{5}$. If $w_{2}$ and $w_{4}$ are both adjacent to $v_{5}$, then the arcs corresponding to $w_{2}, w_{4}$ and $v_{5}$ cover the circle. Otherwise, suppose $w_{2}$ is adjacent to $v_{5}$ and $w_{4}$ is not (the other case is symmetric), so by the circular-arc representation $w_{2}$ belongs to $M_{3}$, and it is adjacent to $w_{3}$.

In this case $w_{2}$ is a twin of $v_{3}$ in $H$. Consider the hole formed by $\left\{v_{1}, v_{2}, w_{2}, v_{4}, v_{5}\right\}$ in $H$, say $C^{\prime}$. In $G,\left\{v_{1}, v_{2}, w_{2}, v_{4}, v_{5}\right\}$ induces a cycle with two chords, $v_{2} v_{4}$ and $w_{2} v_{5}$. If vertex $w_{3}$ has only two neighbors in $C$, then it has two neighbors in $C^{\prime}$, namely $w_{2}$ and $v_{4}$, and it is non-adjacent to $v_{2}$ and $v_{5}$, so we get a contradiction by a previous case (Case 3.3.1).

The last case is when $w_{3}$ has three neighbors in $C$ and $w_{1}$ has only two. If $w_{3}$ belongs to $M_{4}$ then $w_{3}$ and $v_{4}$ are twins in $H$, but the cycle of $H$ obtained by replacing $v_{4}$ with $w_{3}$ in $C$ has only one chord in $G$, contrary to the choice of $C$.

If $w_{3}$ does not belong to $M_{4}$, let $w_{5}$ be a vertex of $M_{4}$, that minimizes the distance of the endpoint of its corresponding arc that lies $A$-between $a_{3}$ and $a_{4}$, to $a_{4}$. Since none of $w_{2}, v_{3}, w_{3}$ belongs to $M_{4}$, they are not adjacent to $w_{5}$. The set of neighbors of $w_{5}$ in $C$ includes $\left\{v_{4}, v_{5}\right\}$ and, by Lemma 19, is a subset of $\left\{v_{1}, v_{2}, v_{4}, v_{5}\right\}$. If $w_{5}$ is adjacent to $v_{1}$ and $v_{2}$, then the arcs corresponding to vertices $v_{2}, v_{4}$ and $w_{5}$ cover the circle. If $w_{5}$ is adjacent to $v_{1}$ but not to $v_{2}$, then the vertices $v_{1}, w_{4}, w_{2}, v_{4}, w_{5}$ induce $C_{5}$. If $w_{5}$ has only two neighbors in $C\left(v_{4}\right.$ and $\left.v_{5}\right)$, then $w_{1}$ and $w_{5}$ are non-adjacent, because $w_{1}$ is non-adjacent to $v_{5}$ and $w_{5}$ is non-adjacent to $v_{1}$. Now if $w_{4}$ and $w_{1}$ are non-adjacent, then the vertices $\left\{v_{1}, \ldots, v_{5}, w_{1}, \ldots, w_{5}\right\}$ induce $T_{2}$, otherwise, the eight vertices $v_{1}, w_{4}, v_{3}, v_{4}, v_{5}, w_{1}, w_{2}, w_{5}$ induce $S_{2}$.

Case $k \geq 3$ : Let $M_{1}, \ldots, M_{2 k+1}$ be the cliques of $H$ such that $M_{1}$ contains 
$v_{1}$ and $v_{2}, M_{2}$ contains $v_{2}$ and $v_{3}, \ldots, M_{2 k+1}$ contains $v_{2 k+1}$ and $v_{1}$, for $1 \leq i \leq 2 k+1, a_{i}$ is an anchor of $M_{i}$, and the vertices corresponding to $M_{1}, M_{2}, \ldots, M_{2 k+1}$ induce $C_{2 k+1}$ in $K(G)$. Let $A=\left\{a_{1}, \ldots, a_{2 k+1}\right\}$. We remind the reader that if $v_{i}$ is adjacent to $v_{j}$ in $G$, then $|i-j| \leq 2$.

If $N=0$, then $G$ contains an odd hole, one of the forbidden subgraphs of Figure 2. If $N=1$, say $v_{1} v_{3}$ is a chord of $C$, then the arcs corresponding to $v_{1}$ and $v_{3}$ intersect in some point of the circle that is $A$-between $a_{1}$ and $a_{2}$. The vertices $v_{1}, v_{2}$ and $v_{3}$ belong to some clique $M$ of $G$, distinct from $M_{i}$ for $i=1, \ldots, 2 k+1$. Every anchor of $M$ is $A$-between $a_{1}$ and $a_{2}$, every vertex of $M$ which is not in $H$ is only adjacent to vertices of $H$ belonging to $M_{1}$ or $M_{2}$ (their corresponding arcs are bounded by $a_{1}$ and $a_{2}$ ), and every vertex of $M$ in $H$ belongs to $M_{1}$ or $M_{2}$. Both $M_{1}$ and $M_{2}$ are disjoint from $M_{4}, \ldots, M_{2 k}$, so $M$ is disjoint from $M_{4}, \ldots, M_{2 k}$. But the vertex $v_{1}$ belongs to $M \cap M_{2 k+1}$ and vertex $v_{3}$ belongs to $M \cap M_{3}$, and therefore $M, M_{3}, M_{4}, \ldots, M_{2 k}, M_{2 k+1}$ induce $C_{2 k}$ in $K(G)$.

Repeating this argument twice (we do not use the fact that the cycle is odd, but only the fact that it has at least six vertices), if there exist two chords $v_{i} v_{i+2}$ and $v_{j} v_{j+2}$ in $C$ such that $v_{i} v_{i+1}, v_{i+1} v_{i+2}, v_{j} v_{j+1}$ and $v_{j+1} v_{j+2}$ are four distinct edges of $G$, we can reduce the problem to a smaller one, the case of an odd hole with $2 k-1$ vertices induced in $K(G)$.

So we only need to consider two cases:

- $N=1$; and

- $N=2$, and for some $i, v_{i}$ is adjacent to $v_{i+2}$ and $v_{i+1}$ is adjacent to $v_{i+3}$.

1. $\underline{N=1}$ : Suppose that the vertices $v_{1}$ and $v_{3}$ are adjacent in $G$. As $v_{3}$ does not belong to $M_{1}$, there is a vertex $w_{1}$ in $M_{1}$ which is not adjacent to $v_{3}$. Analogously, there is a vertex $w_{2}$ in $M_{2}$ which is not adjacent to $v_{1}$. The vertices $w_{1}$ and $w_{2}$ are non-adjacent, otherwise $\left\{v_{1}, v_{3}, w_{2}, w_{1}, v_{2}\right\}$ induces a 4 -wheel, contrary to Lemma 15. By Lemma 19 the vertex $w_{1}$ has two, three or four neighbors in $C$ and they are consecutive in it $\left(v_{2}\right.$ and $v_{1}$; or $v_{2}, v_{1}$ and $v_{2 k+1}$; or $v_{2}, v_{1}, v_{2 k+1}$ and $v_{2 k}$, respectively). Analogously, $w_{2}$ has two, three or four neighbors in $C$ and they are consecutive in the cycle $\left(v_{2}\right.$ and $v_{3}$; or $v_{2}, v_{3}$ and $v_{4}$; or $v_{2}, v_{3}, v_{4}$ and $v_{5}$, respectively). In all cases $w_{1}$ and $w_{2}$ have no common neighbors in $V(C) \backslash\left\{v_{2}\right\}$, since $k \geq 3$.

1.1. If $w_{1}$ and $w_{2}$ have exactly two neighbors each one in $C$, the vertices $v_{1}, \ldots, v_{2 k+1}, w_{1}, w_{2}$ induce a viking.

1.2. If $w_{1}$ and $w_{2}$ have exactly four neighbors each one in $C$, the vertices $w_{1}, v_{2}, w_{2}, v_{5}, \ldots, v_{2 k}$ induce $C_{2 k-1}$.

1.3. If one of $w_{1}, w_{2}$ has exactly three neighbors in $C$ (suppose $w_{1}$, the other case is symmetric), the vertices $w_{1}, v_{2}, v_{3}, \ldots, v_{2 k+1}$ induce $C_{2 k+1}$. 
1.4. If one of $w_{1}, w_{2}$ has exactly two neighbors in $C$ and the other one has exactly four neighbors in $C$, suppose $w_{1}$ has two and $w_{2}$ has four (the other case is symmetric). The clique $M_{4}$ is disjoint from $M_{2}$, so $w_{2}$ does not belong to $M_{4}$ and there is a vertex $w_{3}$ in $M_{4}$ which is not adjacent to $w_{2}$.

The arc corresponding to $w_{3}$ cannot pass through the points of the circle corresponding either to $M_{3}$ (because $w_{2}$ and $w_{3}$ are not adjacent) or to $M_{6}$ (because $M_{4}$ and $M_{6}$ are disjoint), so if the arcs corresponding to $w_{3}$ and $v_{3}$ have non-empty intersection, they must intersect at a point of the circle that is $A$-between $a_{3}$ and $a_{4}$. In this case one of them passes through a point that belongs to both the arc corresponding to $v_{5}$ and the arc corresponding to $w_{2}$, but $w_{3}$ is non-adjacent to $w_{2}$, and $v_{3}$ is non-adjacent to $v_{5}$. So $w_{3}$ and $v_{3}$ are not adjacent, and, by Lemma 19, $w_{3}$ can be adjacent either to $v_{4}, v_{5}, v_{6}$ and $v_{7}$; or to $v_{4}, v_{5}$ and $v_{6}$; or only to $v_{4}$ and $v_{5}$. In the first case, the vertices $v_{1}, v_{3}, v_{4}, w_{3}, v_{7}, \ldots, v_{2 k+1}$ induce $C_{2 k-1}$. In the second case, the vertices $v_{1}, v_{2}, w_{2}, v_{4}, w_{3}, v_{6}, \ldots, v_{2 k+1}$ induce $C_{2 k+1}$. In the last case, the $2 k+4$ vertices $v_{1}, \ldots, v_{2 k+1}, w_{1}, w_{2}, w_{3}$ induce $S_{k}$.

2. $\mathrm{N}=2$, and for some $i, v_{i}$ is adjacent to $v_{i+2}$ and $v_{i+1}$ is adjacent to $v_{i+3}$ :

Without loss of generality, we may assume that $i=1$, so the chords are $v_{1} v_{3}$ and $v_{2} v_{4}$. As $v_{3}$ does not belong to $M_{1}$, there is a vertex $w_{1}$ in $M_{1}$ which is not adjacent to $v_{3}$. As $v_{2}$ does not belong to $M_{3}$, there is a vertex $w_{3}$ in $M_{3}$ which is not adjacent to $v_{2}$. No vertex of $G$ belongs to more than two cliques of $M_{1}, \ldots, M_{2 k+1}$. These facts imply that the vertices $w_{1}$ and $w_{3}$ are non-adjacent, and, by Lemma 19, each of them has two, three or four consecutive neighbors in $C$. The vertex $w_{3}$ can be adjacent to $v_{3}, v_{4}$, $v_{5}$ and $v_{6}$; or to $v_{3}, v_{4}$ and $v_{5}$; or only to $v_{3}$ and $v_{4}$. The vertex $w_{1}$ can be adjacent to $v_{2}, v_{1}, v_{2 k+1}$ and $v_{2 k}$; or to $v_{2}, v_{1}$ and $v_{2 k+1}$; or only to $v_{2}$ and $v_{1}$.

2.1. If $w_{3}$ has four neighbors in $C$, then the vertices $v_{1}, v_{3}, w_{3}, v_{6}, \ldots, v_{2 k+1}$ induce $C_{2 k-1}$. The case of $w_{1}$ having four neighbors is symmetric.

2.2. If $w_{1}$ and $w_{3}$ have three neighbors each one in $C$, then the vertices $w_{1}$, $v_{2}, v_{3}, w_{3}, v_{5}, \ldots, v_{2 k+1}$ induce $C_{2 k+1}$.

2.3. It remains to analyze the cases when $w_{1}$ and $w_{3}$ each have two neighbors in $C$, and when one of them has three neighbors in $C$ and the other one has two. For these cases, we have to consider the clique $M_{2}$.

Since $v_{1}$ and $v_{4}$ do not belong to $M_{2}$, there is a vertex in $M_{2}$ which is not adjacent to $v_{1}$ and there is a vertex in $M_{2}$ which is not adjacent to $v_{4}$.

2.3.1. If there is a vertex $w \in M_{2}$ which is non-adjacent to $v_{1}$ and $v_{4}$, then $w$ is non-adjacent to $w_{1}$ and $w_{3}$, for otherwise $\left\{v_{1}, v_{3}, w\right.$, $\left.w_{1}, v_{2}\right\}$ (or $\left\{v_{2}, w, w_{3}, v_{4}, v_{3}\right\}$, respectively) induces a 4-wheel, contrary to Lemma 15.

Therefore, if $w_{1}$ and $w_{3}$ have two neighbors each in $C$, then 
the vertices $v_{1}, \ldots, v_{2 k+1}, w_{1}, w, w_{3}$ induce a 2-viking in $G$. If $w_{1}$ and $w_{3}$ have two and three neighbors (respectively) in $C$, then $v_{1}, v_{2}, v_{3}, w_{3}, v_{5}, \ldots, v_{2 k+1}, w_{1}, w$ induce a viking in $G$. If $w_{1}$ has three neighbors and $w_{3}$ has two neighbors in $C$, then $w_{1}, v_{2}, v_{3}, \ldots, v_{2 k+1}, w, w_{3}$ induce a viking in $G$.

2.3.2. If no such a vertex $w$ exists, then every vertex of $M_{2}$ is either adjacent to $v_{1}$ or to $v_{4}$, and there exist two vertices $w_{2}$ and $w_{4}$ in $M_{2}$, such that $w_{2}$ is adjacent to $v_{4}$ but not to $v_{1}$ and $w_{4}$ is adjacent to $v_{1}$ but not to $v_{4}$. Since $G$ does not contain a 4 -wheel, it follows that $w_{2}$ is not adjacent to $w_{1}$ and $w_{4}$ is not adjacent to $w_{3}$. If $w_{4}$ is not adjacent to $v_{2 k+1}$ and $w_{2}$ is not adjacent to $v_{5}$, then the vertices $v_{1}, w_{4}, w_{2}, v_{4}, \ldots, v_{2 k+1}$ induce $C_{2 k+1}$. If $w_{4}$ is adjacent to $v_{2 k+1}$ and $w_{2}$ is adjacent to $v_{5}$, then the vertices $w_{4}, w_{2}, v_{5}, \ldots, v_{2 k+1}$ induce $C_{2 k-1}$. Otherwise, suppose $w_{2}$ is adjacent to $v_{5}$ and $w_{4}$ is not adjacent to $v_{2 k+1}$ (the other case is symmetric), so since $G$ is a circular-arc graph, $w_{2}$ belongs to $M_{3}$, and it is adjacent to $w_{3}$. In this case $w_{2}$ is a twin of $v_{3}$ in $H$. Consider the hole $\left\{v_{1}, v_{2}, w_{2}, v_{4}, \ldots, v_{2 k+1}\right\}$, say $C^{\prime}$, in $H$. The graph induced by $\left\{v_{1}, v_{2}, w_{2}, v_{4}, \ldots, v_{2 k+1}\right\}$ in $G$ is a cycle with two chords, $v_{2} v_{4}$ and $w_{2} v_{5}$. If the vertex $w_{3}$ has exactly two neighbors in $C$, then it has exactly two neighbors in $C^{\prime}$, namely $w_{2}$ and $v_{4}$, and it is non-adjacent to $v_{2}$ and $v_{5}$, and we get a contradiction by a previous case (Case 2.3.1).

The last case is when $w_{3}$ has three neighbors in the cycle and $w_{1}$ has only two. If $w_{3}$ belongs to $M_{4}$ then $w_{3}$ and $v_{4}$ are twins in $H$, but the cycle of $H$ obtained by replacing $v_{4}$ with $w_{3}$ in $C$ has only one chord in $G$, contrary to the choice of $C$.

If $w_{3}$ does not belong to $M_{4}$, let $w_{5}$ be a vertex of $M_{4}$, that minimizes the distance of the endpoint of its corresponding arc that lies $A$-between $a_{3}$ and $a_{4}$, to $a_{4}$. Since $w_{2}, v_{3}, w_{3}$ do not belong to $M_{4}$, they are not adjacent to $w_{5}$. The neighbor set of the vertex $w_{5}$ includes $\left\{v_{4}, v_{5}\right\}$ and, by Lemma 19, is a subset of $\left\{v_{4}, v_{5}, v_{6}, v_{7}\right\}$. If $w_{5}$ is adjacent to $v_{6}$ and $v_{7}$, then the vertices $v_{1}, v_{3}, v_{4}, w_{5}, v_{7}, \ldots, v_{2 k+1}$ induce $C_{2 k-1}$. If $w_{5}$ is adjacent to $v_{6}$ but not to $v_{7}$, then the vertices $v_{1}, w_{4}, w_{2}, v_{4}, w_{5}, v_{6}, \ldots, v_{2 k+1}$ induce $C_{2 k+1}$. So we may assume that $v_{4}$ and $v_{5}$ are the only neighbors of $w_{5}$ in $C$. But now, if $w_{4}$ and $w_{1}$ are not adjacent, then the vertices $v_{1}, \ldots, v_{2 k+1}, w_{1}, \ldots, w_{5}$ induce $T_{k}$, and otherwise, the $2 k+4$ vertices $v_{1}, w_{4}, v_{3}, \ldots, v_{2 k+1}, w_{1}, w_{2}, w_{5}$ induce $S_{k}$.

In each case we get a contradiction. This concludes the proof.

We can now prove the characterization theorem for $H C A$ graphs. 
Proof of Theorem 9. The "only if" part follows from Theorem 1, Proposition 2 and Proposition 3. Let us prove the "if" statement. Let $G$ be an $H C A$ graph which does not contain any of the graphs in Figure 2, and let $\mathcal{A}$ be an $H C A$ representation of $G$. Since the class of $H C A$ graph is hereditary, it is enough to prove that $\tau_{c}(G)=\alpha_{c}(G)$.

Assume first that some two arcs of $\mathcal{A}$ cover the circle. Then $\tau_{c}(G) \leq 2$. If $\tau_{c}(G)=1$ or $\alpha_{c}(G)=2$, then $\alpha_{c}(G)=\tau_{c}(G)$ and the theorem holds. So we may assume that $\tau_{c}(G)=2$ and $\alpha_{c}(G)=1$. By Theorem $4, G$ contains $Q_{2 k+1}$ for some $k \geq 1$. It is not difficult to check that the 3 -pyramid is not an $H C A$ graph. Moreover, $\overline{C_{2 k+1}}$ (an induced subgraph of $Q_{2 k+1}$ ) contains the 3 -pyramid for $k \geq 4$. So, $G$ contains either $Q_{3}$, or $Q_{5}$, or $Q_{7}$. But $Q_{3}$ is the 3-sun, $Q_{5}$ contains $C_{5}$ and $Q_{7}$ contains $\overline{C_{7}}$, a contradiction.

So we may assume that no two arcs of $\mathcal{A}$ cover the circle. But now, by Lemma 14 and Theorem 20, $G$ is clique-Helly and K-perfect, and so, by Lemma 10, $\tau_{c}(G)=\alpha_{c}(G)$.

It is easy to check that no two graphs of the families represented in Figure 2 are properly contained in each other. Therefore, as a corollary of Theorem 9, we obtain the following result.

Corollary 21 Vikings, 2-vikings, $S_{k}$ and $T_{k}(k \geq 2)$, are minimally cliqueimperfect.

\subsection{Recognition algorithm}

Helly circular-arc graphs can be recognized in polynomial time [15] and, given a Helly representation of an $H C A$ graph $G$, both parameters $\tau_{c}(G)$ and $\alpha_{c}(G)$ can be computed in linear time $[13,14]$. However, these properties do not immediately imply the existence of a polynomial time recognition algorithm for clique-perfect $H C A$ graphs (we need the equality for every induced subgraph). The characterization in Theorem 9 leads to such an algorithm, which is strongly based on the recognition of perfect graphs [10]. The idea of the algorithm is similar to the one used in [12] for recognizing balanceable matrices.

\section{Algorithm:}

Input: An $H C A$ graph $G=(V, E)$.

Output: TRUE if $G$ is clique-perfect, FALSE if $G$ is not.

(1) Check if $G$ contains a 3 -sun. If $G$ contains a 3 -sun, return FALSE.

(2) (Checking for odd holes and $\overline{C_{7}}$ ) Check if $G$ is perfect. If $G$ is not perfect, return FALSE. 
(3) (Checking for vikings) For every 7-tuple $a_{1}, a_{2}, a_{3}, a_{4}, a_{5}, b_{1}, b_{2}$ such that the edges between those vertices in $G$ are $a_{1} a_{2}, a_{2} a_{3}, a_{2} a_{4}, a_{3} a_{4}, a_{4} a_{5}$, $b_{1} a_{2}, b_{1} a_{3}, b_{2} a_{3}, b_{2} a_{4}$, and possibly $a_{1} a_{5}$, do the following:

(a) If $a_{1}$ is adjacent to $a_{5}$, return FALSE.

(b) Let $G^{\prime}$ be the graph obtained from $G$ by removing the vertices $a_{2}$, $a_{3}, a_{4}, b_{1}, b_{2}$ and all their neighbors except for $a_{1}$ and $a_{5}$, and adding a new vertex $c$ adjacent only to $a_{1}$ and $a_{5}$.

(c) Check if $G^{\prime}$ is perfect. If $G^{\prime}$ is not perfect, return FALSE.

(4) (Checking for 2-vikings) For every 8-tuple $a_{1}, a_{2}, a_{3}, a_{4}, a_{5}, b_{1}, b_{2}, b_{3}$ such that the edges between those vertices in $G$ are $a_{1} a_{2}, a_{2} a_{3}, a_{2} a_{4}, a_{3} a_{4}, a_{3} a_{5}$, $a_{4} a_{5}, b_{1} a_{2}, b_{1} a_{3}, b_{2} a_{3}, b_{2} a_{4}, b_{3} a_{4}$ and $b_{3} a_{5}$, do the following:

(a) If $a_{1}$ is adjacent to $a_{5}$, return FALSE.

(b) Let $G^{\prime}$ be the graph obtained from $G$ by removing the vertices $a_{2}, a_{3}$, $a_{4}, b_{1}, b_{2}, b_{3}$ and all their neighbors except for $a_{1}$ and $a_{5}$, and adding a new vertex $c$ adjacent only to $a_{1}$ and $a_{5}$.

(c) Check if $G^{\prime}$ is perfect. If $G^{\prime}$ is not perfect, return FALSE.

(5) (Checking for $S_{k}$ ) For every 8-tuple $a_{1}, a_{2}, a_{3}, a_{4}, a_{5}, b_{1}, b_{2}, b_{3}$ such that the edges between those vertices in $G$ are $a_{1} a_{2}, a_{2} a_{3}, a_{3} a_{4}, a_{3} a_{5}, a_{4} a_{5}, b_{1} a_{1}$, $b_{1} a_{2}, b_{2} a_{4}, b_{2} a_{5}, b_{3} a_{1}, b_{3} a_{2}, b_{3} a_{3}, b_{3} a_{4}$, and possibly $a_{1} a_{5}$, do the following:

(a) If $a_{1}$ is adjacent to $a_{5}$, return FALSE.

(b) Let $G^{\prime}$ be the graph obtained from $G$ by removing the vertices $a_{2}, a_{3}$, $a_{4}, b_{1}, b_{2}, b_{3}$ and all their neighbors except for $a_{1}$ and $a_{5}$, and adding a new vertex $c$ adjacent only to $a_{1}$ and $a_{5}$.

(c) Check if $G^{\prime}$ is perfect. If $G^{\prime}$ is not perfect, return FALSE.

(6) (Checking for $T_{k}$ ) For every 10-tuple $a_{1}, a_{2}, a_{3}, a_{4}, a_{5}, b_{1}, b_{2}, b_{3}, b_{4}, b_{5}$ such that the edges between those vertices in $G$ are $a_{1} a_{2}, a_{2} a_{3}, a_{2} a_{4}, a_{3} a_{4}$, $a_{3} a_{5}, a_{4} a_{5}, b_{1} a_{1}, b_{1} a_{2}, b_{2} a_{1}, b_{2} a_{2}, b_{2} a_{3}, b_{2} b_{3}, b_{3} a_{1}, b_{3} a_{2}, b_{3} a_{3}, b_{3} a_{4}, b_{3} b_{4}$, $b_{4} a_{3}, b_{4} a_{4}, b_{4} a_{5}, b_{5} a_{4}, b_{5} a_{5}$, and possibly $a_{1} a_{5}$, do the following:

(a) If $a_{1}$ is adjacent to $a_{5}$, return FALSE.

(b) Let $G^{\prime}$ be the graph obtained from $G$ by removing the vertices $a_{2}$, $a_{3}, a_{4}, b_{1}, b_{2}, b_{3}, b_{4}, b_{5}$ and all their neighbors except for $a_{1}$ and $a_{5}$, and adding a new vertex $c$ adjacent only to $a_{1}$ and $a_{5}$.

(c) Check if $G^{\prime}$ is perfect. If $G^{\prime}$ is not perfect, return FALSE.

(7) Return True.

Correctness: The output of the algorithm is TRUE if it finishes in step (7), otherwise the output is FALSE. Let us prove that, given as input an $H C A$ graph $G$, the algorithm finishes in step (7) if and only if $G$ does not contain the graphs of Figure 2. The correctness of the algorithm then follows from Theorem 9.

Let $G$ be an $H C A$ graph. Step (1) will output FALSE if and only if $G$ contains a 3 -sun. So henceforth suppose that $G$ does not contain a 3 -sun.

1. Step (2) will output FALSE if and only if $G$ contains an odd hole or $\overline{C_{7}}$. 
If $G$ contains an odd hole or $\overline{C_{7}}$ then it is not perfect. Conversely, if $G$ is not perfect it contains an odd hole or an odd antihole. Since $G$ is $H C A$, it does not contain an antihole of length at least nine. So $G$ must contain an odd hole or $\overline{C_{7}}$. This proves 1 . So henceforth suppose that $G$ is perfect, and, in particular, it does not contain an odd hole or $\overline{C_{7}}$.

2. Step (3) will output FALSE if and only if $G$ contains a viking.

If $G$ contains a viking $H$ with $V(H)=\left\{a_{1}, \ldots, a_{2 k+1}, b_{1}, b_{2}\right\}$ and adjacencies as defined in Section 2, at some point the algorithm will consider the 7-tuple $a_{1}, a_{2}, a_{3}, a_{4}, a_{5}, b_{1}, b_{2}$. In $H$, either $k=2$ and $a_{1}$ is adjacent to $a_{5}$ (in this case the algorithm will output FALSE at step (3.a)) or $a_{5}$ and $a_{1}$ are joined by an odd path of length at least three, $a_{5} a_{6} \ldots a_{2 k+1} a_{1}$. Since $a_{6}, \ldots, a_{2 k+1}$ are nonneighbors of $a_{2}, a_{3}, a_{4}, b_{1}, b_{2}$, it follows that $c a_{5} a_{6} \ldots a_{2 k+1} a_{1} c$ is an odd hole in $G^{\prime}$, so the algorithm will output FALSE at step (3.c).

Conversely, if the algorithm outputs FALSE at step (3.a), then $\left\{a_{1}, \ldots, a_{5}\right.$, $\left.b_{1}, b_{2}\right\}$ induce a viking in $G$. If the algorithm outputs FALSE at step (3.c), then $G^{\prime}$ is not perfect. Since at this point we are assuming that $G$ is perfect, the vertex $c$ must belong to an odd hole or odd antihole in $G^{\prime}$. Since it has degree two, $c$ belongs to an odd hole $c a_{5} v_{1} \ldots v_{2 t} a_{1} c$ in $G^{\prime}$. Since $v_{1}, \ldots, v_{2 t}$ are different from and non-adjacent to $a_{2}, a_{3}, a_{4}, b_{1}, b_{2}$, it follows that $\left\{a_{1}, \ldots, a_{5}\right.$, $\left.v_{1}, \ldots, v_{2 t}, b_{1}, b_{2}\right\}$ induce a viking in $G$. This proves 2 . So henceforth suppose that $G$ contains no viking.

3. Step (4) will output FALSE if and only if $G$ contains a 2-viking.

If $G$ contains a 2-viking $H$ with $V(H)=\left\{a_{1}, \ldots, a_{2 k+1}, b_{1}, b_{2}, b_{3}\right\}$ and adjacencies as defined in Section 2, at some point the algorithm will consider the 8-tuple $a_{1}, a_{2}, a_{3}, a_{4}, a_{5}, b_{1}, b_{2}, b_{3}$. In $H$, either $k=2$ and $a_{1}$ is adjacent to $a_{5}$ (in this case the algorithm will output FALSE at step (4.a)) or $a_{5}$ and $a_{1}$ are joined by an odd path of length at least three, $a_{5} a_{6} \ldots a_{2 k+1} a_{1}$. Since $a_{6}, \ldots, a_{2 k+1}$ are non-neighbors of $a_{2}, a_{3}, a_{4}, b_{1}, b_{2}, b_{3}$, it follows that $c a_{5} a_{6} \ldots a_{2 k+1} a_{1} c$ is an odd hole in $G^{\prime}$, so the algorithm will output FALSE at step (4.c).

Conversely, if the algorithm outputs FALSE at step (4.a), then $\left\{a_{1}, \ldots, a_{5}\right.$, $\left.b_{1}, b_{2}, b_{3}\right\}$ induce a 2 -viking in $G$. If the algorithm outputs FALSE at step (4.c), then $G^{\prime}$ is not perfect. Since at this point we are assuming that $G$ is perfect, the vertex $c$ must belong to an odd hole or odd antihole in $G^{\prime}$. Since it has degree two, $c$ belongs to an odd hole $c a_{5} v_{1} \ldots v_{2 t} a_{1} c$ in $G^{\prime}$. Since $v_{1}, \ldots, v_{2 t}$ are different from and non-adjacent to $a_{2}, a_{3}, a_{4}, b_{1}, b_{2}, b_{3}$, it follows that $a_{1}, \ldots, a_{5}, v_{1}, \ldots, v_{2 t}, b_{1}, b_{2}, b_{3}$ induce a 2 -viking in $G$. This proves 3 . So henceforth suppose that $G$ contains no 2-viking.

4. Step (5) will output FALSE if and only if $G$ contains $S_{k}$ for some $k \geq 2$. 
If $G$ contains $S_{k}$ for some $k \geq 2$, with $V\left(S_{k}\right)=\left\{a_{1}, \ldots, a_{2 k+1}, b_{1}, b_{2}, b_{3}\right\}$ and adjacencies as defined in Section 2 , at some point the algorithm will consider the 8-tuple $a_{1}, a_{2}, a_{3}, a_{4}, a_{5}, b_{1}, b_{2}, b_{3}$. In $S_{k}$, either $k=2$ and $a_{1}$ is adjacent to $a_{5}$ (in this case the algorithm will output FALSE at step (5.a)) or $a_{5}$ and $a_{1}$ are joined by an odd path of length at least three, $a_{5} a_{6} \ldots a_{2 k+1} a_{1}$. Since $a_{6}, \ldots, a_{2 k+1}$ are non-neighbors of $a_{2}, a_{3}, a_{4}, b_{1}, b_{2}, b_{3}$, it follows that $c a_{5} a_{6} \ldots a_{2 k+1} a_{1} c$ is an odd hole in $G^{\prime}$, so the algorithm will output FALSE at step (5.c).

Conversely, if the algorithm outputs FALSE at step (5.a), then vertices $\left\{a_{1}\right.$, $\left.\ldots, a_{5}, b_{1}, b_{2}, b_{3}\right\}$ induce $S_{2}$ in $G$. If the algorithm outputs FALSE at step (5.c), then $G^{\prime}$ is not perfect. Since at this point we are assuming that $G$ is perfect, the vertex $c$ must belong to an odd hole or odd antihole in $G^{\prime}$. Since it has degree two, $c$ belongs to an odd hole $c a_{5} v_{1} \ldots v_{2 t} a_{1} c$ in $G^{\prime}$. Since $v_{1}, \ldots, v_{2 t}$ are different from and non-adjacent to $a_{2}, a_{3}, a_{4}, b_{1}, b_{2}, b_{3}$, it follows that vertices $\left\{a_{1}, \ldots, a_{5}, v_{1}, \ldots, v_{2 t}, b_{1}, b_{2}, b_{3}\right\}$ induce $S_{t+2}$ in $G$. This proves 4 . So henceforth suppose that $G$ does not contain $S_{k}$ for $k \geq 2$.

5. Step (6) will output FALSE if and only if $G$ contains $T_{k}$ for some $k \geq 2$.

If $G$ contains $T_{k}$ for some $k \geq 2$, with $V\left(T_{k}\right)=\left\{a_{1}, \ldots, a_{2 k+1}, b_{1}, b_{2}, b_{3}, b_{4}, b_{5}\right\}$ and adjacencies as defined in Section 2 , at some point the algorithm will consider the 10-tuple $a_{1}, \ldots, a_{5}, b_{1}, \ldots, b_{5}$. In $T_{k}$, either $k=2$ and $a_{1}$ is adjacent to $a_{5}$ (in this case the algorithm will output FALSE at step (6.a)) or $a_{5}$ and $a_{1}$ are joined by an odd path of length at least three, $a_{5} a_{6} \ldots a_{2 k+1} a_{1}$. Since $a_{6}, \ldots, a_{2 k+1}$ are non-neighbors of $a_{2}, a_{3}, a_{4}, b_{1}, b_{2}, b_{3}$, it follows that $c a_{5} a_{6} \ldots a_{2 k+1} a_{1} c$ is an odd hole in $G^{\prime}$, so the algorithm will output FALSE at step (6.c).

Conversely, if the algorithm outputs FALSE at step (6.a), then vertices $\left\{a_{1}, \ldots, a_{5}, b_{1}, \ldots, b_{5}\right\}$ induce $S_{2}$ in $G$. If the algorithm outputs FALSE at step (6.c), then $G^{\prime}$ is not perfect. Since at this point we are assuming that $G$ is perfect, the vertex $c$ must belong to an odd hole or odd antihole in $G^{\prime}$. Since it has degree two, $c$ belongs to an odd hole $c a_{5} v_{1} \ldots v_{2 t} a_{1} c$ in $G^{\prime}$. Since $v_{1}, \ldots, v_{2 t}$ are different from and non-adjacent to $a_{2}, a_{3}, a_{4}, b_{1}, \ldots, b_{5}$, it follows that $\left\{a_{1}, \ldots, a_{5}, v_{1}, \ldots, v_{2 t}, b_{1}, \ldots, b_{5}\right\}$ induce $T_{t+2}$ in $G$. This proves 5 , and completes the proof of correctness.

Time complexity: The time complexity of the best known algorithm to recognize perfect graphs is $O\left(|V|^{9}\right)[10]$. So the time complexity of this algorithm is given by step $(6)$ and it is $O\left(|V|^{19}\right)$.

Thus we can answer affirmatively the question of the existence of a polynomial time recognition algorithm for clique-perfect graphs within the class of $H C A$ graphs. 


\section{Summary}

These results allow us to formulate partial characterizations of clique-perfect graphs by forbidden subgraphs, as is shown in Table 1.

\begin{tabular}{|l|l|l|}
\hline Graph classes & Forbidden subgraphs & Reference \\
\hline \hline Diamond-free graphs & odd generalized suns & Thm 8 \\
\hline$H C A$ graphs & graphs in Figure 2 & Thm 9 \\
\hline
\end{tabular}

Table 1

Forbidden induced subgraphs for clique-perfect graphs in each studied class.

Note that in the second case all the forbidden induced subgraphs are minimal. In the first case, however, we need to forbid every odd-generalized sun. Obviously, in this case it is enough to forbid diamond-free odd generalized suns. It is easy to see that all such suns have no improper edges but we do not yet know what the minimal diamond-free odd generalized suns are.

\section{References}

[1] V. Balachandhran, P. Nagavamsi, and C. Pandu Rangan, Clique-transversal and clique-independence on comparability graphs, Information Processing Letters 58 (1996), 181-184.

[2] H. Bandelt and E. Prisner, Clique graphs and Helly graphs, Journal of Combinatorial Theory. Series B 51 (1991), 34-45.

[3] C. Berge and M. Las Vergnas, Sur un théorème du type König pour hypergraphes, Annals of the New York Academy of Sciences 175 (1970), 32-40.

[4] F. Bonomo, M. Chudnovsky, and G. Durán, Partial characterizations of cliqueperfect graphs, Electronic Notes in Discrete Mathematics 19 (2005), 95-101.

[5] F. Bonomo, M. Chudnovsky, and G. Durán, Partial characterizations of cliqueperfect graphs I: sublcasses of claw-free graphs, submitted to Discrete Applied Mathematics (2005).

[6] F. Bonomo and G. Durán, Characterization and recognition of Helly circulararc clique-perfect graphs, Electronic Notes in Discrete Mathematics 22 (2005), $147-150$.

[7] F. Bonomo, G. Durán, M. Groshaus, and J. Szwarcfiter, On clique-perfect and K-perfect graphs, Ars Combinatoria, to appear.

[8] A. Brandstädt, V. Chepoi, and F. Dragan, Clique $r$-domination and clique $r$-packing problems on dually chordal graphs, SIAM Journal on Discrete Mathematics 10 (1997), 109-127. 
[9] L. Chong-Keang and P. Yee-Hock, On graphs without multicliqual edges, Journal of Graph Theory 5 (1981), 443-451.

[10] M. Chudnovsky, G. Cornuéjols, X. Liu, P. Seymour, and K. Vušković, Recognizing Berge Graphs, Combinatorica 25 (2005), 143-187.

[11] M. Chudnovsky, N. Robertson, P. Seymour, and R. Thomas, The Strong Perfect Graph Theorem, Annals of Mathematics, to appear.

[12] M. Conforti and G. Zambelli, Recognizing Balanceable Matrices, Mathematical Programming. Series B, to appear.

[13] G. Durán, M. Lin, S. Mera, and J. Szwarcfiter, Clique-independent sets on Helly circular-arc graphs, Electronic Notes in Discrete Mathematics 18 (2004), 103-108.

[14] G. Durán, M. Lin, S. Mera, and J. Szwarcfiter, Algorithms for finding cliquetransversals of graphs, submitted to Annals of Operations Research (2005).

[15] F. Gavril, Algorithms on circular-arc graphs, Networks 4 (1974), 357-369.

[16] V. Guruswami and C. Pandu Rangan, Algorithmic aspects of clique-transversal and clique-independent sets, Discrete Applied Mathematics 100 (2000), 183202.

[17] J. Lehel and Z. Tuza, Neighborhood perfect graphs, Discrete Mathematics 61 (1986), 93-101.

[18] C. Lucchesi, C. Picinin de Mello, and J. Szwarcfiter, On clique-complete graphs, Discrete Mathematics 183 (1998), 247-254.

[19] E. Prisner, Hereditary clique-Helly graphs, The Journal of Combinatorial Mathematics and Combinatorial Computing 14 (1993), 216-220. 